\title{
Giant Fusiform Vertebral Artery Aneurysm Treated with Microsurgical Bypass and Endovascular Coiling after Failed Flow Diversion: A Case Report and Discussion
}

\author{
Steven H. Cook ${ }^{1}$ Timothy D. Miller $^{1} \quad$ L. Fernando Gonzalez ${ }^{1} \quad$ Ali R. Zomorodi $^{1}$ \\ ${ }^{1}$ Department of Surgery, Division of Neurosurgery, Duke University \\ Medical Center, Durham, North Carolina, United States \\ Address for correspondence Steven H. Cook, MD, Duke University \\ Medical Center, DUMC Box 3807, Durham, NC 27710, United States \\ (e-mail: steven.cook@dm.duke.edu).
} Indian J Neurosurg 2016;5:202-206

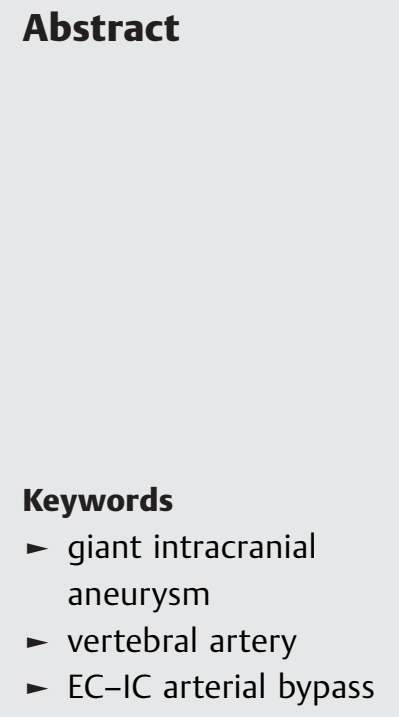

Background Giant vertebral and vertebrobasilar aneurysms have proven to be extremely challenging vascular entities to treat despite many advances in microsurgical and endovascular techniques. In recent years, there has been more attention paid to combined approaches, including combinations of endovascular and surgical procedures in hopes of decreasing the associated morbidity and mortality of these lesions.

Clinical Presentation We present a case of a giant fusiform vertebral artery aneurysm that was initially treated with flow-diverting stents. After the failure of flow diversion to occlude the aneurysm, the lesion was definitively treated with an occipital artery to the posterior inferior cerebellar artery (PICA) bypass and coil embolization of residual aneurysm.

Conclusion After bypass and parent vessel deconstruction, angiography confirmed the aneurysm has remained occluded and the PICA patent. This case highlights the use of an extracranial-to-intracranial bypass to salvage a complex residual aneurysm after failed flow diversion.

\section{Background and Importance}

Giant vertebral and vertebrobasilar (VB) aneurysms constitute a rare form of intracranial aneurysms that exhibit a dismal natural history associated with significant morbidity and mortality. ${ }^{1}$ Current endovascular treatments include coil embolization as well as the more recent use of flow-diverting stents, each having known risks of aneurysm recurrence, ischemia, and death. ${ }^{2}$ Extracranial-intracranial (EC-IC) bypasses have been used to grant adequate perfusion for parent vessel deconstruction or flow reversal. We present a case of a giant partially thrombosed fusiform aneurysm of the distal left vertebral artery treated with a flow-diverting stent and rescued, after its failure, with microsurgical bypass and coil embolization.

received

March 24, 2015

accepted after revision

December 23, 2015

published online

June 8, 2016
DOI http://dx.doi.org/ 10.1055/s-0036-1581984. ISSN 2277-954X.

\section{Clinical Presentation}

A 37-year-old patient presented with gait ataxia to a local neurosurgeon's office. Brain imaging revealed a large left vertebral aneurysm, $3 \mathrm{~cm}$ in diameter and $6 \mathrm{~cm}$ in length, with clinical and radiographical compression on the lower brainstem ( - Fig. 1). A cerebral angiogram confirmed a giant, partially thrombosed fusiform aneurysm of the distal left V4 segment. The left posterior inferior cerebellar artery (PICA) originated from the aneurysm.

The decision was made by the initial treating surgeon to explore surgically, aiming toward primary clip reconstruction of the aneurysm through an extended petrous exposure. The aneurysm was too calcified for adequate clip placement. Due to failure of primarily clip

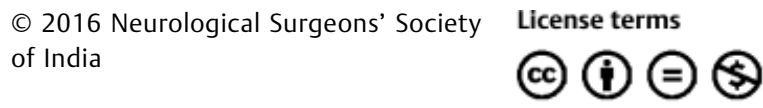




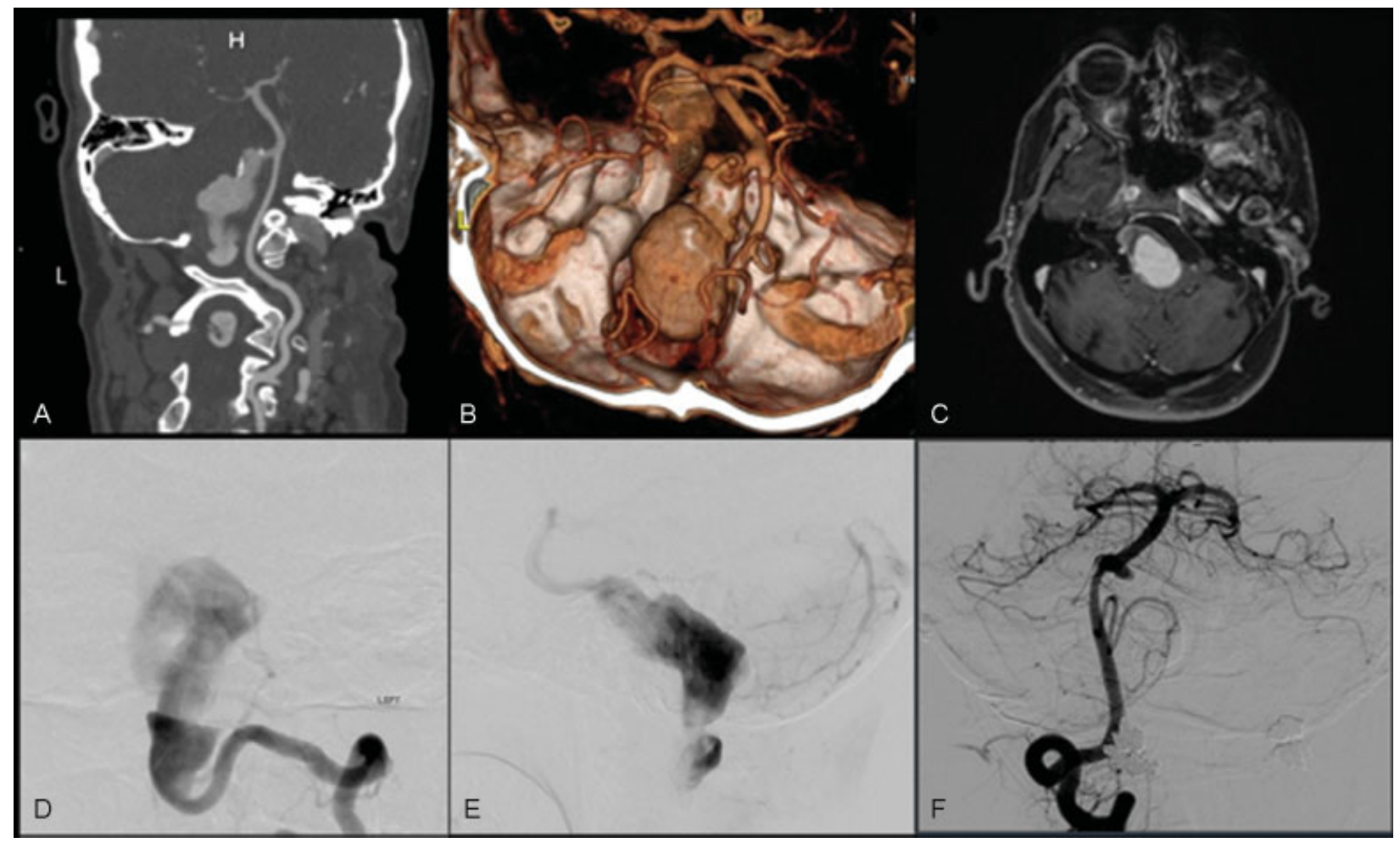

Fig. 1 (A) CTA of large fusiform left vertebral artery aneurysm. (B) 3D reformat of CTA. (C) Axial MRI with contrast showing partially thrombosed aneurysm. (D and E) Left vertebral artery angiogram. (F) Right vertebral artery angiogram. 3D, three-dimensional; CTA, computed tomographic angiography; MRI, magnetic resonance imaging.

reconstruction, the treating team chose to pursue an endovascular option.

\section{Endovascular Treatment}

The cerebral angiogram showed a patent right vertebral artery with the filling of the distal aspect of the aneurysm. There was a minimal flow through the thrombosed portion of the aneurysm precluding stent placement in the left vertebral artery. On serial angiograms, the distal portion of the aneurysm was enlarged at the VB junction. Flow diversion within the right vertebral artery was performed with a $3.25 \mathrm{~mm} \times 16 \mathrm{~mm}$ Pipeline stent (Covidien Neurovascular, Irvine, California, United States) from the proximal basilar artery to the right vertebral artery.

A 3-month follow-up angiogram showed residual filling within distal portions of the left vertebral aneurysm and an enlarging proximal portion. The left PICA remained patent and the stented right vertebral artery showed adequate filling of the posterior circulation. Given this recurrence, the patient had a telescoped $3.5 \mathrm{~mm} \times 16 \mathrm{~mm}$ Pipeline stent placed to isolate the distal portion of the aneurysm. Coil embolization was performed for the proximal filling through a left vertebral approach, ensuring that the left PICA remained patent.

A 6-month follow-up angiogram showed no filling of the aneurysm from the right side; however, the proximal portion had increased in size. The left PICA filled from the blind sac and therefore coil embolization was not performed. At this point, given failed aneurysm occlusion by flow diversion, the patient was referred to the authors for definitive treatment. Given the need to preserve the left PICA and previous flow diversion of the contralateral vertebral artery preventing a direct occlusion of the parent vessel, an occipital artery (OA) to PICA bypass was planned to be followed by coil occlusion of the remaining pouch.

\section{Bypass Procedure}

The patient was placed in a left lateral position with a lumbar drain. The left OA was traced with percutaneous Doppler and dissected from surrounding tissue. A combined far lateral approach, with a partial mastoidectomy and condylectomy, allowed exposure of the suboccipital triangle and the extracranial portion of the left vertebral artery.

The dura was opened and the left PICA tonsillar segment was trapped with two temporary clips in preparation for the bypass. The distal OA was prepared for anastomosis and a temporary clip was placed proximally. End-to-side OA to PICA anastomosis was performed with 10-0 interrupted sutures and clips removed. Flow was confirmed with Doppler monitoring. A cranioplasty was performed that allowed a relaxed conduit for the arterial anastomosis. The wound was closed in layers with a loose closure of the dura to maximize flow through the anastomosis. Total operative time was 410 minutes and the blood loss was $300 \mathrm{~mL}$.

\section{Postoperative Course and Embolization}

After an arteriogram demonstrated adequate flow through the bypass, coil embolization of the proximal aneurysmal 


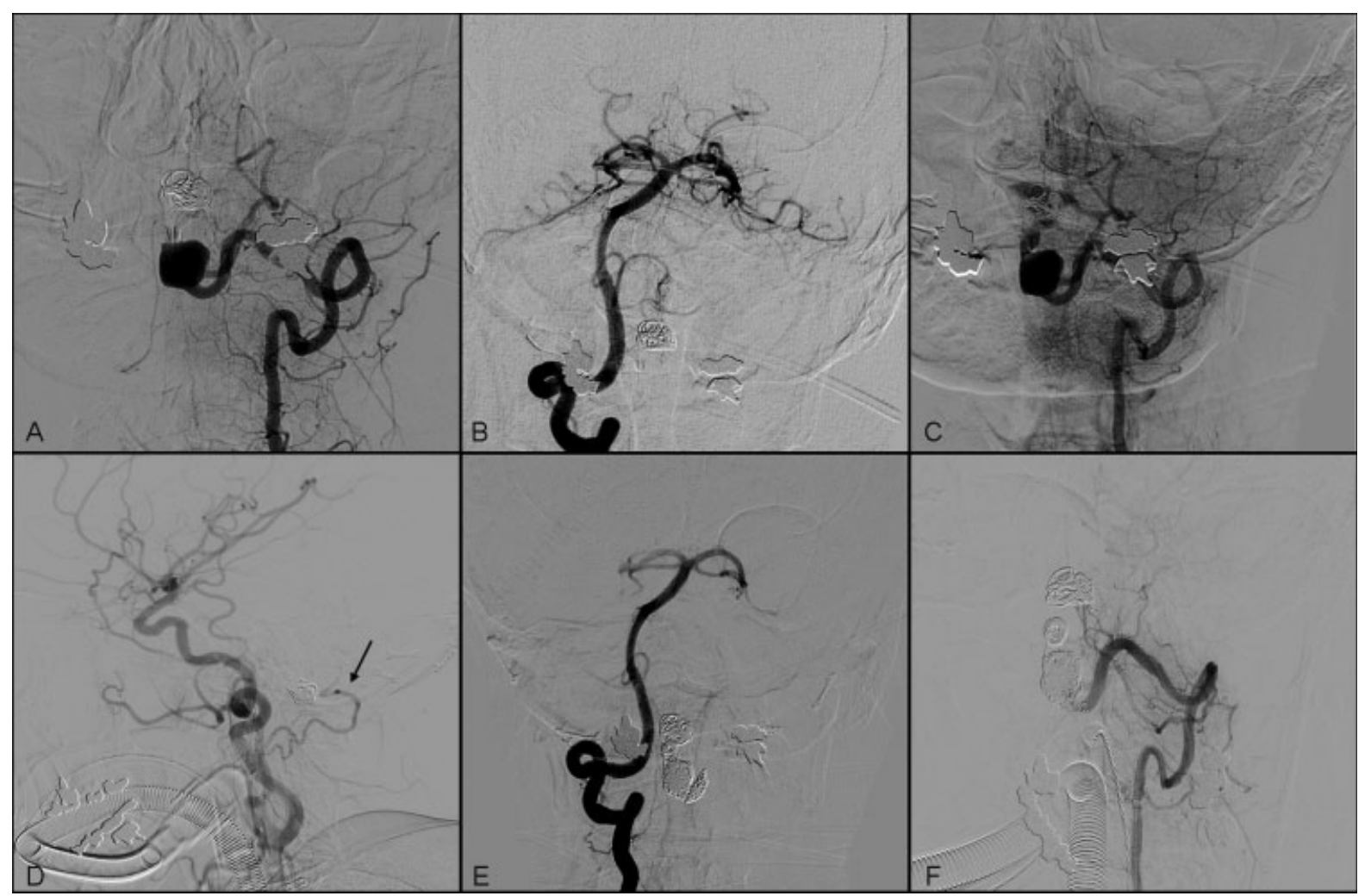

Fig. 2 (A) Left VA angiogram with partial coiling of aneurysm, (B) right VA angiogram after stent placement. (C) Postcoiling showing residual aneurysm with PICA origin inclusion, (D) left carotid angiogram showing patent OA-PICA, (E) 6 month postoperative angiogram-right VA. (F) 6 month post-op angiogram - left vertebral artery with no residual aneurysm. PICA, posterior inferior cerebellar artery; OA, occipital artery; VA, vertebral artery.

pouch was performed. There were no complications postoperatively. The patient had an uneventful hospital course throughout remaining at preoperative neurological baseline and was safely discharged home on antiplatelet therapy. Angiography performed at 6 months after the final procedure showed no residual aneurysm and a patent left occipital-PICA anastomosis (-Fig. 2).

\section{Discussion}

The use of flow diversion in this particular case is arguable and the benefit was none. The increasingly widespread use of these devices can create more complicated cases requiring definitive treatment. The best known utility of flow diversion in $V B$ junction aneurysms occurs in lesions containing a saccular component. The natural history of these aneurysms results in death in $80 \%$ of untreated patients and even with treatment, there is significant associated neurologic morbidity, hemorrhage, and death. ${ }^{1}$ The historic treatments of ligation and clip reconstruction have not significantly changed these outcomes. Endovascular therapy is more commonly used today, but is more difficult to perform in vertebral artery fusiform aneurysms with recurrence rates of coiling as high as $40 \%{ }^{3}$

Recent studies using microsurgical bypass have demonstrated stabilization of lesions in patients having good-to-moderate outcomes on average 60 to $65 \%$ of the time. ${ }^{4,5}$ The specific architecture of the aneurysm determines if the lesion is amenable to endovascular occlusion (i.e., coil, stent coil, or flow diversion). Proximal occlusion or ligation in the presented case without further vascular bypass, runs a significant risk of infarction due to the reliance on the anterior circulation and the contralateral vertebral artery in the setting of previously placed flowdiverting stents.

\section{Endovascular Therapy}

With coil embolization, consideration should be given to the need for parent artery preservation, the likelihood of aneurysm recurrence, and impact of coils on surrounding structures. Stent-assisted coiling methods have allowed for preservation of blood flow in fusiform aneurysms but present a risk of migration and perforating vessel occlusion. Wakhloo et al found higher aneurysm recanalization rates in the posterior circulation when compared with anterior circulation after stent or stentassisted coiling (33 vs. $6 \%$, respectively). ${ }^{6}$ Other reported combined mortality and recurrence rates have been reported in the $40 \%$ range. ${ }^{7}$

Use of new flow-diverting stents as a primary treatment has had a variable benefit in the treatment of posterior circulation lesions. Siddiqui et al showed in seven patients that two had a good outcome following placement. ${ }^{2}$ Ertl et al showed in a series of six patients: two early-stent occlusions, 
four brainstem ischemic events, and four deaths after a mean follow-up of 13 months. $^{8}$ In contrast, Chalouhi et al showed occlusion or significant reduction in posterior circulation aneurysms in over $80 \%$ of patients using the Pipeline device at 6 months in a series of six patients. ${ }^{9}$ Monteith et al demonstrated in a series of 24 fusiform aneurysms that $>95 \%$ occlusion occurred in almost $60 \%$ of the patients. There were no major procedural complications and at follow-up over $80 \%$ were clinically stable or improved with one death reported from hemorrhage. ${ }^{10}$ In the treatment of posterior circulation aneurysms with stenting, studies have identified persistent compression, primarily brainstem, as a source of morbidity. ${ }^{11}$ After endovascular therapy, persistent or new compression of surrounding structures from aneurysm thrombus or growth may require open surgical decompression and possible surgical bypass. $^{12}$

\section{Surgical Bypass}

Proximal ligation can carry a significant morbidity as a stand-alone treatment. With the origin of PICA within the aneurysm (as in this case) and concern that flow may be diminished through the flow-diverting stent placed previously across the VB junction, there is a significant concern for ischemia in PICA distribution. Surgical clipping has become the standard treatment for these lesions. In some cases, clip ligation is not feasible due to the configuration of the aneurysm, calcification, and involvement of perforating vessels. In the event that endovascular treatment is not an option, then vascular bypass remains a viable treatment. Depending on the location of aneurysms and vascular territories from parent arteries, a low- versus high-flow bypass can be performed. In cases of VB aneurysms, donor options for a direct bypass include the superficial temporal artery (STA) and OA while the recipient sites vary by level of vessel occlusion but the posterior cerebral artery (PCA), superior cerebellar artery (SCA), and PICA are the most common. High-flow bypasses may also be performed with saphenous vein or radial artery grafts. There are numerous approaches that may be utilized depending on the segment of the basilar artery that must be exposed. Exposure relies on skull-based principles of maximizing bone resection and minimizing brain retraction and includes variations of retrosigmoid and far lateral approaches. ${ }^{13}$

Recent case series have shown improved outcomes with grafting. Kalani et al showed good-to-moderate outcomes at long-term follow-up in 54\% of the patients using low-flow bypasses from STA to various vessels. ${ }^{4}$ Shi et al had a large series of posterior circulation aneurysms, which included VB and PICA origin aneurysms. They conducted bypass in 14 patients with over $90 \%$ graft patency seen on follow-up and good outcomes in all OA to PICA bypasses performed. ${ }^{14}$

With an OA to PICA bypass, preoperative evaluation should include assessing the adequate size of the OA $(>0.8$ $\mathrm{mm}$ ), adequate diameter of recipient PICA within area without perforators, course identified for an adequate length needed, and secondary plan in case of damage to the OA. Technical considerations include safe dissection of the $\mathrm{OA}$, avoidance of vasospasm in the $\mathrm{OA}$, and adequate closure to prevent artery occlusion and cerebrospinal fluid leak. $^{15}$

\section{Conclusion}

The present report presents the use of EC to IC bypass and parent vessel deconstruction to rescue a case of failed flow diversion. The use of flow diversion for fusiform aneurysms does not provide homogeneous predictable results. In today's neurosurgical armamentarium, there is a wide diversity of devices and techniques that can be used to provide a definitive treatment for cerebral aneurysms.

\section{Conflict of Interest}

There are no conflicts of interest or financial disclosures to report. All authors approve this article for submission.

\section{References}

1 Drake CG, Peerless SJ. Giant fusiform intracranial aneurysms: review of 120 patients treated surgically from 1965 to 1992. J Neurosurg 1997;87(2):141-162

2 Siddiqui AH, Abla AA, Kan P, et al. Panacea or problem: flow diverters in the treatment of symptomatic large or giant fusiform vertebrobasilar aneurysms. J Neurosurg 2012;116(6): 1258-1266

3 Henkes H, Fischer S, Mariushi W, et al. Angiographic and clinical results in 316 coil-treated basilar artery bifurcation aneurysms. J Neurosurg 2005;103(6):990-999

4 Kalani MYS, Zabramski JM, Nakaji P, Spetzler RF. Bypass and flow reduction for complex basilar and vertebrobasilar junction aneurysms. Neurosurgery 2013;72(5):763-775, discussion 775-776

5 Miyamoto S, Funaki T, Iihara K, Takahashi JC. Successful obliteration and shrinkage of giant partially thrombosed basilar artery aneurysms through a tailored flow reduction strategy with bypass surgery. J Neurosurg 2011;114(4):1028-1036

6 Wakhloo AK, Mandell J, Gounis MJ, et al. Stent-assisted reconstructive endovascular repair of cranial fusiform atherosclerotic and dissecting aneurysms: long-term clinical and angiographic follow-up. Stroke 2008;39(12):3288-3296

7 Chen Z, Yang Y, Miao H, et al. Endovascular treatment for large and giant fusiform aneurysms of the vertebrobasilar arteries. Clin Imaging 2013;37(2):227-231

8 Ertl L, Holtmannspötter M, Patzig M, Brückmann H, Fesl G. Use of flow-diverting devices in fusiform vertebrobasilar giant aneurysms: a report on periprocedural course and long-term follow-up. AJNR Am J Neuroradiol 2014;35(7):1346-1352

9 Chalouhi N, Tjoumakaris S, Dumont AS, et al. Treatment of posterior circulation aneurysms with the pipeline embolization device. Neurosurgery 2013;72(6):883-889

10 Monteith SJ, Tsimpas A, Dumont AS, et al. Endovascular treatment of fusiform cerebral aneurysms with the Pipeline Embolization Device. J Neurosurg 2014;120(4):945-954

11 Meckel S, McAuliffe W, Fiorella D, et al. Endovascular treatment of complex aneurysms at the vertebrobasilar junction with flowdiverting stents: initial experience. Neurosurgery 2013;73(3): 386-394

12 Abla AA, Zaidi HA, Crowley RW, et al. Optic chiasm compression from mass effect and thrombus formation following unsuccessful 
treatment of a giant supraclinoid ICA aneurysm with the Pipeline device: open surgical bailout with STA-MCA bypass and parent vessel occlusion. J Neurosurg Pediatr 2014;14(1):31-37

13 Bambakidis NC, Gonzalez LF, Amin-Hanjani S, et al. Combined skull base approaches to the posterior fossa. Technical note. Neurosurg Focus 2005;19(2):E8
14 Shi X, Qian H, Singh KC, et al. Surgical management of vertebral and basilar artery aneurysms: a single center experience in 41 patients. Acta Neurochir (Wien) 2013;155(6):1087-1093

15 Nossek E, Chalif DJ, Dehdashti AR. How I do it: occipital artery to posterior inferior cerebellar artery bypass. Acta Neurochir (Wien) 2014;156(5):971-975 